\title{
The Functional Significance of Human Notochord in the Development of Vertebral Golumn. An Electron Microscopic Study
}

\author{
Takashi Murakami, ${ }^{*} \dagger$ Eikichi Wakamatsu, ${ }^{*}$ Nobuaki \\ Tamahashi $\dagger$ and Tohru Takahashi $\dagger$ \\ *Department of Orthopaedics, Tohoku University School of. \\ Medicine, Sendai 980 and $\dagger$ Department of Pathology, the \\ Research Institute for Tuberculosis and Cancer, Tohoku \\ University, Sendai 980
}

\begin{abstract}
Murakami, T., Wakamatsu, E., Tamahashi, N. and Takahashi, T. The Functional Significance of Human Notochord in the Development of Vertebral Column. An Electron Microscopic Study. Tohoku J. exp. Med., 1985, 146(3), 321-336 — Twenty-one human embryos or fetuses from four to twenty-one weeks in gestation age were submitted to light and electron microscopic observations of the notochord and its related structures. The notochord cells were found to have densely developed rER and Golgi areas, which were correlated with an activity to secrete into their surroundings specific ground substance probably containing proteoglycan. The highest differentiation of this cell as estimated by the peak secretory activity was reached at about ten weeks, while signs of degeneration emerged shortly thereafter and became increasingly apparent with the advancing gestation. The synthesis and secretion of matrices were likely to be taken over by the fibrocartilage cells surrounding the notochord by about 20 weeks when the notochord cells had undergone severe degenerative changes. Another characteristic feature of the notochord cells at their fully differentiated stage was abundance in intracytoplasmic glycogen that coexisted with striking paucity of mitochondria. This was interpreted as suggesting the metabolic dependence of these cells upon anaerobic glycolysis even at the peak of their secretory performance.

notochord ; vertebral column ; proteoglycan ; fetal development ; electron microscopy
\end{abstract}

The human notochord appears in the dorsal central portion of embryo as an elongated anlage, plays an important role in forming the vertebral column and later obliterates. So far, the function of this organ has not been clearly explained although it has been assumed by some that the notochord serves as a substantial material for the forthcoming formation of nucleus pulposus of intervertebral discs, until its cells degenerate and are taken over by the surrounding fibrocartilage ; the

Received December 26, 1984 ; accepted for publication March 14, 1985.

Offprint request to : Takashi Murakami, M.D., Department of Orthopaedics, Tohoku University School of Medicine, 1 Seiryomachi, Sendai 980, Japan. 
degeneration of notochord cells has been reported to begin in the sixth gestational month at earliest. However, these reports were based on light microscopic studies alone but not on meticulous cytology. In an effort to establish the development and involution of this organ on more strict morphological basis, we performed electron microscopic analysis of the notochord in human embryos and fetuses ranging from four to twenty-one gestational weeks, which we intend to describe in this article. The change of notochord cell along with the gestational age especially of its subcellular structure was remarkable and gave much insight into the function of human notochord about which, only ambiguous assumption has been made in previous studies.

\section{Material and Methods}

Twenty-one human embryos or fetuses obtained by legal abortion served as the basic material. The gestational age estimated according to the standard of Moore (1977) that is by measuring the crown-rump distance, ranged from four to twenty-one weeks. All these were submitted to electron microscopic studies of the notochord and other anlages of the vertebral column, with additional light microscopic studies. The embryos, i.e., those younger than nine gestational weeks, were all sectioned horizontally for electron microscopy; sections for light microscopy were simultaneously prepared from Epon-embedded blocks. In fetuses older than these, lumbar intervertebral discs were horizontally cut and were examined for electron microscopy, while lower thoracic column was taken for additional light microscopic studies. From the latter sample, a set of horizontal, sagittal and coronal sections were prepared for the sake of 3-dimensional perspective.

For electron microscopy, small pieces of vertebral column were fixed in ice-cold buffered mixture of $2 \%$ glutaraldehyde and $2 \%$ formaldehyde and were post-fixed in $1 \%$ osmium tetroxide. The tissues were embedded in Epon 812 and ultrathin sections were doubly stained with uranyl acetate and lead citrate, which were observed with an electron microscope (Hitachi, H-300). For light microscopic studies of fetal materials, paraffin sections of formalin-fixed tissues were stained by the following methods ; HE, toluidin blue, alcian blue (at pH 1.0 and 2.5), Kossa, periodic-acid Schiff with and without digestion by diastase.

\section{RESULTS}

\section{Light microscopic observations}

So far as the light microscopic aspects are concerned, the development of human notochord has been described in detail (Keys and Compere 1932 ; Peacock 1951; Walmsley 1953; O'Rahilly and Meyer 1979; O'Rahilly et al. 1980). Nevertheless, in the following we intend to present an outline of it not only for the sake of better perspective for the electron micrographs that follow, but on account of some findings the present analysis has confirmed.

At about the fourth week of gestation, the notochord presents as a minute rod slightly flattened dorso-ventrally and running along the midline, keeping an approximately equidistant interposition between the aorta and the overlying neural tube. This rod consists of densely packed notochord cells that are apparently epithelial, has a continuous basal lamina along the surface and is surrounded 
by immature mesenchymal cells, the sclerotome. At the seventh week the matrix around the notochord shows metachromasia to toluidin blue, and at about the same time vertebral rudiments emerge centrifugally surrounding the notochord (Fig. 1). Their matrix, showing similar metachromasia, is much lower in its intensity than in the notochord. The metachromasia of the notochord persists until the seventeenth week while at about twenty weeks the tinctorial property becomes ambiguous.

At about eight weeks the notochord that has been a straight column is split into small globular segments arranged in a row, corresponding to the positions of future intervertebral discs. Fig. 2 shows a sagittal section of a fetus of 11.5 weeks. The segmented notochord remains in the intervertebral spaces as small globules around which a flat immature cartilage already assumes the shape of an intervertebral disc. Between two adjacent discs is formed a primordial vertebral body in which the remnant of notochord remains as a so-called mucoid streak connecting two neighboring notochord globules. At about ten weeks the cytoplasm of notochord cells becomes positive for PAS reaction and persists so until at least twenty-one weeks ; this is labile to digestion by diastase and therefore is attributed to glycogen accumulation. At about the same time, thin matrix faintly positive for alcian blue appears in the intercellular spaces. The future vertebral bodies at this stage are cartilage having a matrix that intensely stains with alcian blue, while its portions contiguous to the notochord stain only weakly, probably because their differentiation is delayed.

At about eleven weeks, the matrix of vertebral anlages begins to calcify at the central portion of bodies (Fig. 2). Blood vessels emerge and begin to invade from the surroundings; these are sinusoid-like vessels with dilated lumina, lined by endothelial layer and surrounded by mesenchymal cells. Interestingly, the vessels extend as if aiming at the primordial vertebral bodies and evading the intervertebral spaces in which there are segmented notochord (Fig. 3), a strikingly regular, but hitherto unnoticed arrangement that is likely to have some inductive effect on the segmentation of vertebral column. Almost simultaneously, the cartilage cells at the central portion of future vertebral bodies begin to involute and undergo vacuolar degeneration, showing pyknotic or karyorrhectic nuclei when enclosed in the calcified zone.

At about thirteen weeks the central portion of the future vertebral bodies comes to be hollowed into primordial medullary cavity. At this stage, bone formation by osteoblasts is apparent with partially decalcified matrices turning to show intense metachromasia to toluidin blue. Growth of blood vessels from the surroundings advances, still concentrating at the level of vertebral bodies. The mucoid streaks remain devoid of columnar cartilage and, of course, ossification is much delayed here; these places stain weakly with alcian blue, just like the matrix of notochord itself.

The place of future nucleus pulposus that has been composed almost exclu- 
sively by notochord cells until the seventeenth week, comes to be replaced gradually by inner cartilage cells (Fig. 4 ).

Fig. 5 is a schema summarizing the development of vertebral column, chiefly referring to the relationship among the notochord, vertebral bodies and blood vessels. The main points are; a straight notochord at 5 weeks, its complete segmentation leaving vestigial mucoid streaks at 9 weeks, beginning calcification of primordial vertebrae and invading blood vessels at 11 weeks and formation of medullary cavity at 13 weeks.

\section{Electron microscopic observation}

The notochord cells. At about five gestational weeks, the individual cells composing a notochord were connected by means of interdigitation, with desmosome-like junctions sparsely placed along the intercellular borders (Fig. 6). The connection of cells was so close as to exclude any room for discernible intercellular spaces. The nucleus at this stage of notochord cells was large, round or ovoid, and devoid of heterochromatin. The cytoplasm was rich in glycogen particles but organelles were only poorly developed with a few mitochondria and endoplasmic reticulum with meager ribosomes, a condition suggesting little, if any, secretory function (Fig. 7). A small number of intervening filaments were found but these did not show any particular localization or direction. The matrix in the vicinity of notochord had a few flocculent filaments to which, at some places, small particles of high density were attached.

In the embryos of seven weeks Golgi apparatus emerged in the cytoplasm and at this stage $\mathrm{rER}$ enclosing mitochondria was readily visible. There were also slightly increased amount of filaments in the cytoplasm, showing partly reticular structure.

At ten to eleven weeks, the intercellular spaces became dilated due to accumulation of matrix that will be described in the following (Fig. 8). The notochord cells were detached one from another by this accumulation and were connected with the adjacent ones at separate points, at least partially at desmosome-like junctions. In the nucleus that had slightly indented contour, heterochromatin appeared as a marginated dense zone under the nuclear membrane. Outside the indented places of nucleus, small coated vesicles emerged. The Golgi complex was also conspicuous and mature in structure. Rough ER was dominant, markedly lamellated, elongated and partially dilated to form cisternae (Fig. 9). The fully developed rER and Golgi complex suggested that the notochord cells at this stage assumed a very active secretory function; this was also evidenced by the increased amount in the intercellular spaces of matrix which was likely to be the secretory product of notochord cells. This, similar to the substance around the notochord of younger embryos, contained reticular network of filaments attached by two sorts of particles, both of which were of about the same size but the one with higher density was assumed to be the macromolecule of 
proteoglycan (Fig. 10). The filaments and particles continued increasing in density until at least the twentieth gestational week. In the cytoplasm, there were massive accumulations of electron-lucent substance; on account of the strong PAS-reaction in the corresponding light microscopy, these clouds were considered to be deposits of glycogen. Focally increased filaments were also seen. Mitochondria appeared much reduced in number, and some were vacuolated or swollen probably reflecting degenerative processes which they had already undergone. A mantle of basement membrane material still lay along the external side of notochord cells, where a small number of micropinocytotic vesicles were seen (Fig. 10.)

In the fetuses of sixteen to seventeen weeks, many villous processes were found protruding from the cell membranes facing intercellular spaces. Rough ER was still dilated containing amorphous material. Mitochondria, at least those with integrated structure, were no longer discernible.

At twenty weeks the nucleus of notochord cells was irregularly shrunken with markedly indented contour, indicating further advanced degeneration (Fig. 11). The heterochromatin was strikingly aggregated. The cytoplasm contained in addition to massive glycogen pools vacuoles of various size that were densely packed at the indented places of nucleus, where some networks of $\mathrm{rER}$ were also seen in contact with these vacuoles. Some notochord cells had bundles of filaments which corresponded in thickness to so-called intermediate filaments and were arranged in an evidently undulated pattern (Fig. 12). The shrinkage of nucleus and the occurrence of both cytoplasmic vacuoles and filaments, that began at about the twelfth week and intensified thereafter, were all regarded as degenerative signs of notochord cells.

The immature cartilage cells. At the stage of four weeks, the immature mesenchymal cells of the sclerotome investing the notochord did not show any differentiation but these were assumed to be immature cartilage cells, judging from their position. The cells were dispersed separately in homogeneous ground substance. The nucleus were relatively large and round and contained only euchromatin. The intracytoplasmic organelles were scanty, showing very sparsely arranged mitochondria, rER or Golgi apparatus (Fig. 13).

At about ten weeks, aggregated heterochromatin appeared along the margin of the nucleus of these immature cells. Rough ER and Golgi area growingly developed. Mitochondria were still hardly discernible (Fig. 14). There were a small amount of filaments in some cells. The matrix contained finely netty filaments, to which particles of high density, probably proteoglycan, were abundantly attached.

In the fetuses of about twenty weeks, the elongation and enlargement of rER were conspicuous. In the adjacent matrix, in addition to the network of filaments, were thick fibers showing periodical striping, probably collagen (Fig. 15). 


\section{Discussion}

So far, the human notochord has generally been assumed to be an anlage of nucleus pulposus of future intervertebral discs, but how and when the notochord degenerates and gives way to the components of mature disc has been only poorly understood. What a part the notochord plays in the formation of disc has also been a matter of ambiguity. Keys and Compere had the impression that even at the time of birth, the notochord continued to serve as the main source supplying the material of nucleus pulposus. Peacock regarded the nucleus pulposus of full-term fetus as still consisting of notochordal tissue. Degenerative changes of notochordal cells, however, were reported by Walmsley to begin at about the 28th week of gestation. On the other hand, Wolfe et al. (1965) claimed that the notochord contribute to the formation of the matrix of nucleus pulposus not only in all fetal life but also in some postnatal days. But Meachim and Roy (1967) hardly saw notochord cells in the postnatal nucleus pulposus which he reported was dispersed by a small number of fibrocartilage-composing cells.

These discrepancies may be at least partially due to the fact that these studies relied exclusively upon light microscopic observations. We analyzed a series of developmental processes of human notochord including its late involution by means of electron microscope, and found that the notochord cell begins to degenerate at an unexpectedly early stage of twelve to sixteen weeks of gestation. This is evidenced not only by such nuclear changes of notochord cells as shrinkage or pyknosis, but also by the gradually lowering level of their once activated secretory function, as clearly visualized by the changes of cellular ultrastructure. The increase of intracytoplasmic filaments with advancing gestation might also reflect aging and degeneration of notochord cell, seeing that it appears to have close bearing with the increase in cytoplasmic vacuoles, change of nuclear chromatin pattern and irregularity of nuclear margin. Increased density of similar filaments was observed in the joint cartilage cell of rabbit (Barnett et al. 1963) and in that of man (Meachim and Roy); in both observations this change was attributed to cartilaginous degeneration. Correlation of these changes with the development of the axial skeleton is of fundamental importance in analyzing the functional implication of notochord.

The notochord cell, sufficiently equipped with rER and Golgi areas, exhibits its differentiation into a secretory apparatus, at least at its fully activated stage. The secretory product that accumulates in the intercellular spaces appears quite likely to contain proteoglycan, as shown by the peculiar fine structure of the matrix that comprises fine filaments forming a network closely associated with dense particles. Equally likely, this matrix appears to increase as the secretory organelles of notochord cells develop. Based on this correlation, the period during which the notochord cell retains its secretory capacity was roughly estimated to range from the seventh to the seventeenth week of gestation. On the 
other hand, signs of degeneration of these cells emerge as early as at the middle of this functional period, as mentioned above.

Malinsky (1956) proved abundant intracytoplasmic glycogen in fetal notochord cells of man. In the present study too, a large amount of glycogen is apparent throughout the period between 5 to 20 weeks, in electron microscopic pictures as well as in strongly positive PAS that is liable to prior diastase digestion. This glycogen may be interpreted as suggesting that notochord cells are rather more dependent on glycolysis than on respiration. Being devoid of penetrating capillaries, the notochord, like cartilaginous tissues, probably requires the constituent cells to adapt to anaerobic circumstances. Wolfe et al. demonstrated in these cells enzyme systems not only for aerobic but anaerobic metabolism. The especially "bradytrophic" character of notochord cells is reflected in the paucity of mitochondria in their cytoplasm. Interestingly, in the period between the 5th and the 10th weeks of gestation during which rER of notochord cells extends greatly its intracytoplasmic surface, mitochondria markedly dwindle in number and density ; it appears likely that the synthesis and secretion of the specific matrices by this cell are mainly performed on the basis of anaerobic metabolism.

The cells of fibrocartilage enclosing the notochord, having scanty cytoplasmic organelles at about ten weeks, thereafter become equipped with growingly dense rER and Golgi areas; the cells at the twentieth week are sufficiently rich in these organelles as to suggest vigorous production of matrix substances. Since, judging from the development of organelles, the secretory capacity of notochord cells is considered to reach a peak at about the tenth week and declines thereafter, it may be reasonable to assume that these cells, though playing some role in the formation of nucleus pulposus in the first half of gestation, are taken over by the fibrocartilage cells in the following period.

The authors examined the process of development and degeneration of human notochord by means of electron microscopy, and showed that notochord cells basically belong to those endowed with remarkable secretory activities. Accordingly, much is expected to contribute to better understanding of the hitherto ambiguous significance of this organ, if further studies are performed on its ultrastructure from the viewpoint of functional morphology. We have resorted to some morphometric methods to analyze more accurately the process of rise and fall of secretory capacity of notochord, but this will be published elsewhere.

\section{References}

1) Barnett, C.H., Cochrane, W. \& Palfrey, A.J. (1963) Age change in articular cartilage of rabbit. Ann. rheum. Dis., 22, 389-400.

2) Keyes, D.C. \& Compere, E.L. (1932) The normal and pathological physiology of the nucleus pulposus of the intervertebral disc. J. Bone Joint Surg., 14, 897-938.

3) Malinsky, J. (1956) Histochemical demonstration of carbohydrates in intervertebral discs of human embryos. Acta histochem. 3, 297-307. 
4) Meachim, G. \& Roy, S. (1967) Intracytoplasmic filaments in the cells of adult human articular cartilage. Ann. rheum. Dis., 26, 50-58.

5) Moore, K.L. (1977) The Developing Human. 2nd ed., Saunders, Philadelphia.

6) O'Rahilly, R. \& Meyer, D.B. (1979) The timing and sequence of events in the development of the human vertebral column during the embryonic period proper. Anat. Embryol., 157, 167-176.

7) O'Rahilly, R., Muller, F. \& Meyer, D.B. (1980) The human vertebral column at the end of the embryonic period proper. 1. The column as a whole. J. Anat., 131, 565-575.

8) Peacock, A. (1951) Observation on the prenatal development of the intervertebral disc in man. J. Anat., 85, 260-274.

9) Walmsley, R. (1953) The development and growth of the intervertebral disc. Edinburgh med. $J$., 60, 341-364.

10) Wolfe, H.J., Putschar, G.J. \& Vickery, A.L. (1965) Role of the notochord in human intervertebral disc. 1. Fetus and infant. Clin. Orthop., 39, 205-212.

Fig. 1. A horizontal section of an embryo of 7 weeks, showing the notochord $(\mathrm{N})$ invested in sclerotome. Seen in the lower part is the spinal cord. Epon section. Toluidin blue. $\times 4$.

Fig. 2. The axial skeleton of a fetus 11.5 weeks, shown by sagittal sectioning. The notochord $(\mathrm{N})$ is split into globules, two neighbors of which are halfway connected with a mucoid streak (arrow). The future discs (D) and vertebral bodies (VB) are clearly molded, the latter subject to enchondral ossification. PAS. $\times 2$.

Fig. 3. Sagittally cut vertebral column of a fetus 13.5 weeks. Small blood vessels (arrows) are extending into vertebral bodies evading the intervertebral disc (D), the upper and the lower borders of which are denoted by dots. $\mathrm{N}$ : notochord. HE. $\times 4$.

Fig. 4. Seventeen gestation weeks. Advancing enchondral ossification of a vertebral body (upper). The notochord $(\mathrm{N})$ is being replaced by cells of inner cartilage. HE. $\times 4$.

Fig. 5. A schema illustrating the early developmental process of vertebral column. 

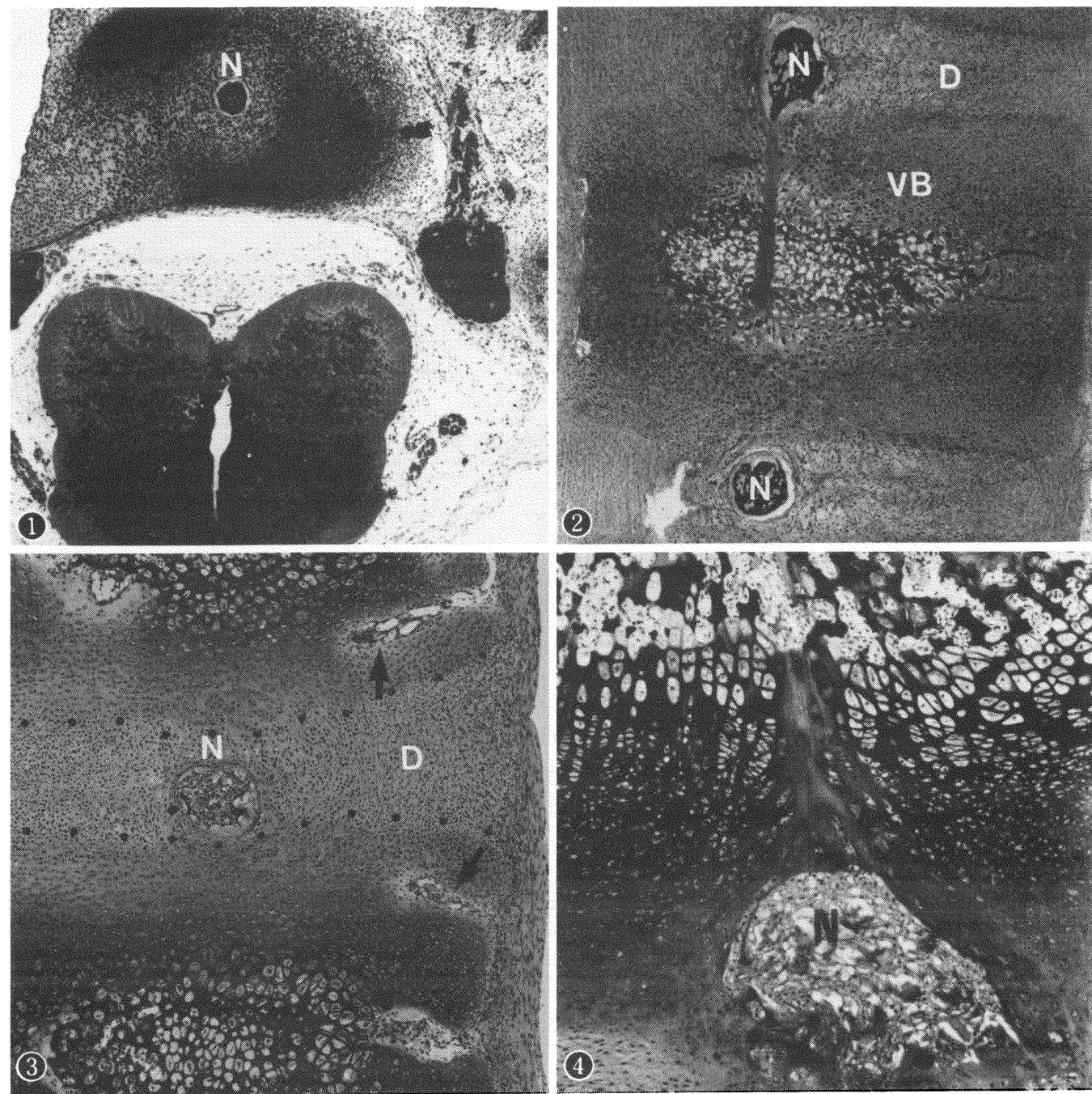

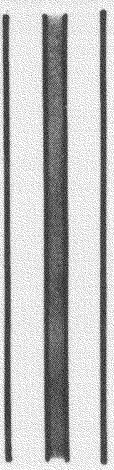

$5 w$

notochord in sclerotome

5

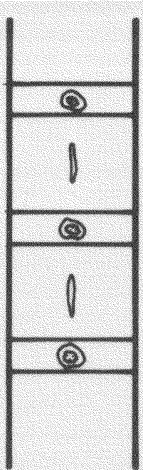

$9 w$

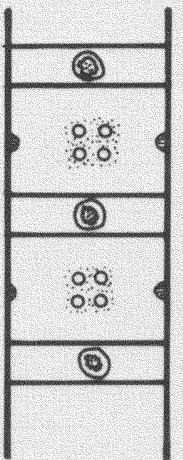

$11 \mathrm{w}$

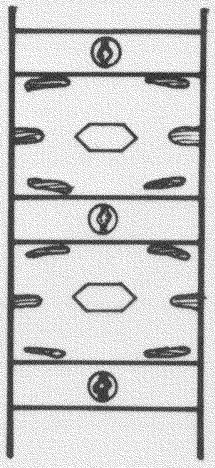

$13 w$ calcification of invasion of matrix budding vessels primordial of vessels medullary cavity 
Fig. 6. An electron micrograph of the notochord at 5 weeks of gestation. In the outer space are immature mesenchymal cells of sclerotome. $\times 1,000$.

Fig. 7. Notochord cells at 4.5 gestational weeks. The cytoplasm rich in glycogen particles (GLY) has poorly developed organelles. There are some areas interlaced by filaments (F). $\times 5,000$. 

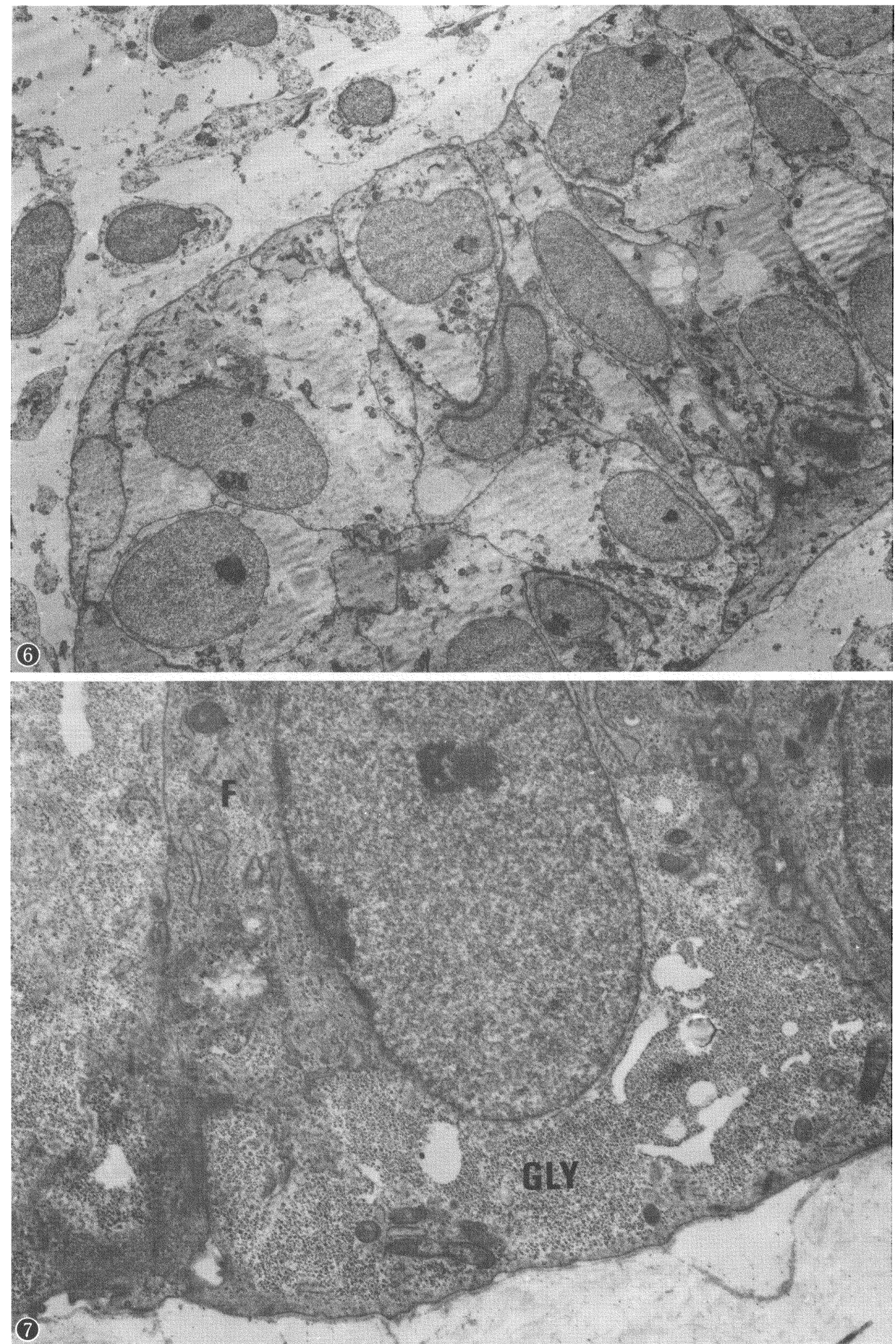
Fig. 8. A notochord cell at 10.5 weeks with intercellular spaces (IS) between the adjacent cells dilated due to accumulation of specific ground substance. Moderately indented nucleus. The amorphous electron-lucent clouds, indicated by arrows, appear to be intracytoplasmic deposits of glycogen. $\times 7,000$.

Fig. 9. A notochord cell at 10.5 weeks. Fully developed rER and Golgi complex (G). Filaments are increased to form thick bundles. $\times 7,000$. 

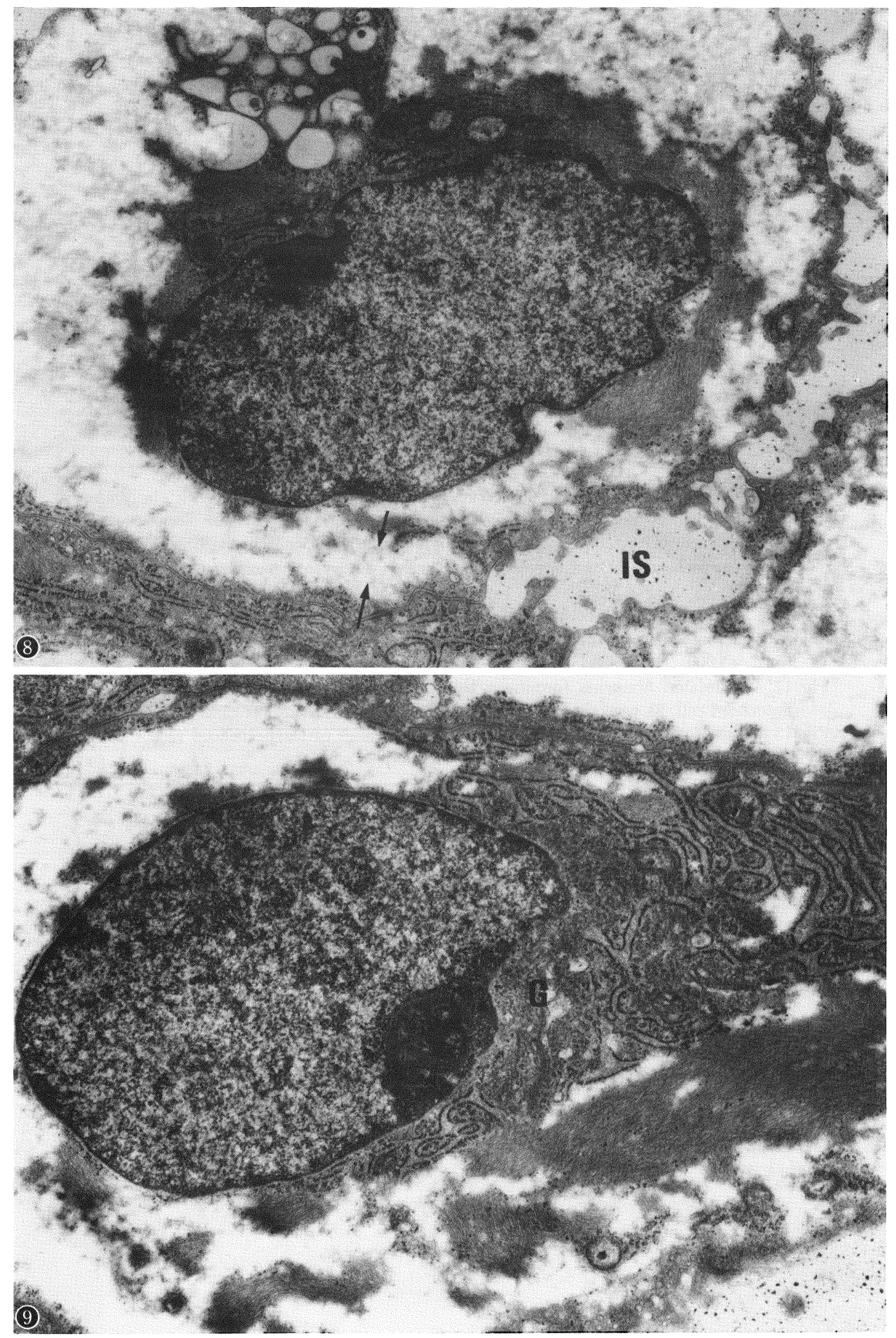
Fig. 10. Superficial portion of a notochord cell, 10.5 weeks, is shown with the ground substance in the outer space. Macromolecules of proteoglycan are seen as dense particles (arrows) attached to fine reticular structure. Beneath the basement membrane (arrowheads) of the notochord cell, many pinocytotic vesicles (V) are formed. $\times 15,000$.

Fig. 11. Two notochordal cells at 21 weeks. Markedly shrunken nucleus, aggregated heterochromatin and abundant cytoplasmic vacuoles, all suggesting advanced degeneration. $\times 5,000$.

Fig. 12. Undulated filaments forming a thick bundle in subplasmalemmal portion of a notochord cell, 21 weeks. Dilated intercellular space in the lower right part. $\times 10,000$.

Fig. 13. Immature mesenchymal cells in the surroundings of the notochord $(\mathrm{N})$, 4 weeks, with poorly developed intracytoplasmic organelles. $\times 2,000$. 


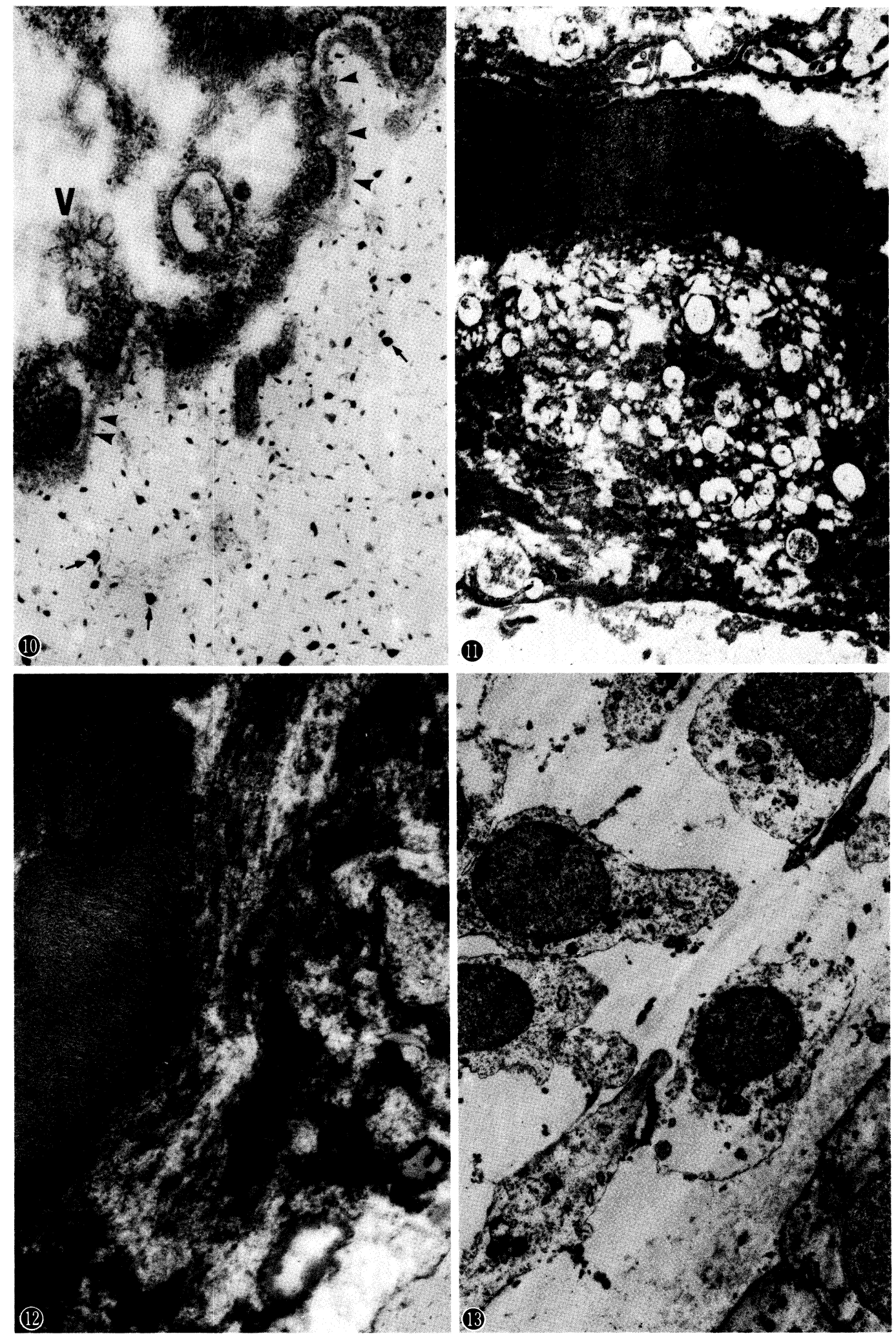



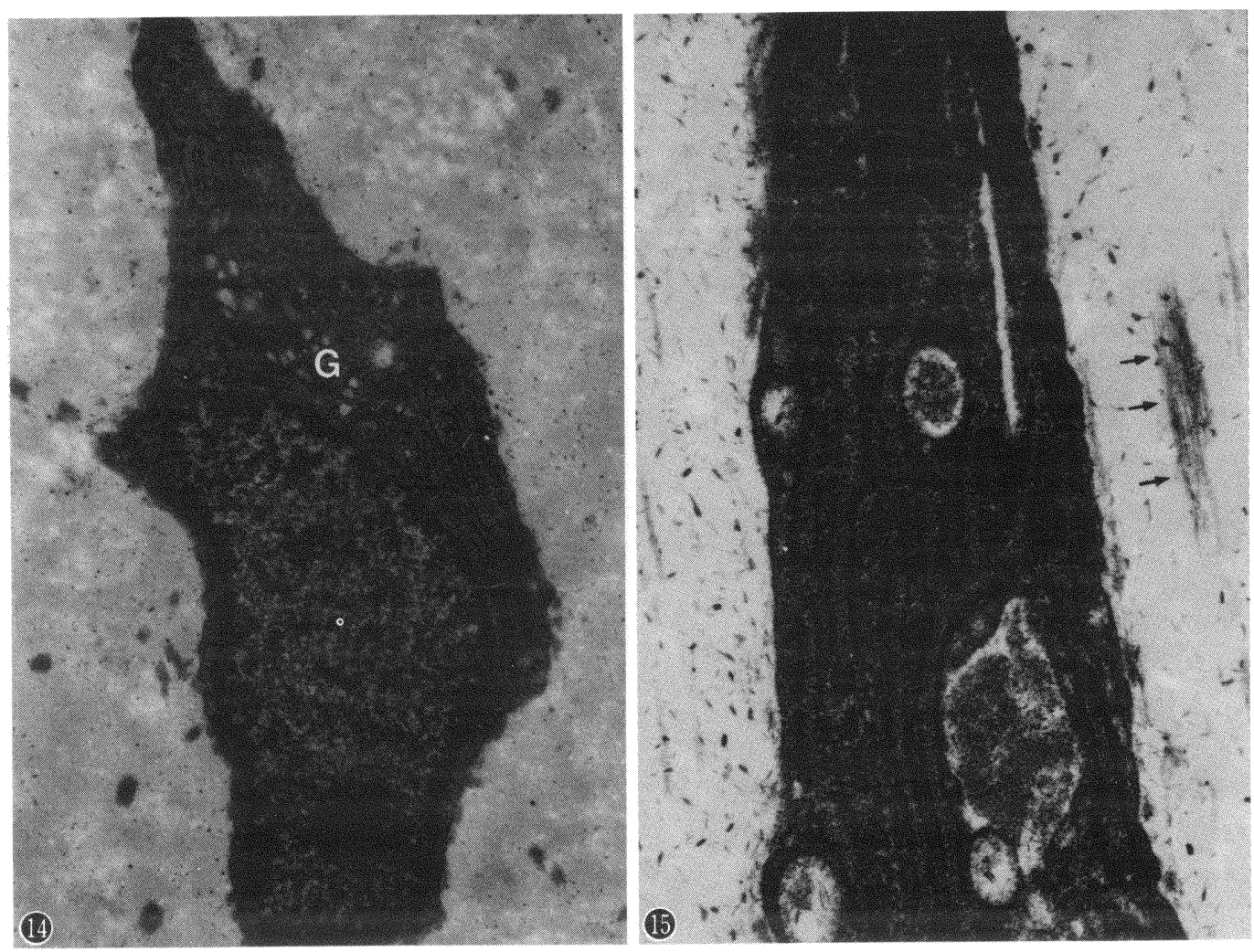

Fig. 14. A fibrocartilage cell at 10 weeks, with cytoplasm rich in rER. Well developed Golgi complex $(G) . \times 5,000$.

Fig. 15. A fibrocartilage cell at 20 weeks having abundant rER. Thick fibers (arrows), probably collagen, are apearing in the ground substance. $\times 15,000$. 